\title{
REFLEXION CRITIQUE SUR LA LOI PORTANT FINANCEMENT DES PARTIS POLITIQUES AU REGARD DES ELECTIONS DE 2011 EN REPUBLIQUE DEMOCRATIQUE DU CONGO.
}

\author{
Par Mumba Kakudji Martial ${ }^{1}$ et Mutonwa Kalombe Jean-Marc Pacifique ${ }^{2}$
}

\section{INTRODUCTION}

Les années de dictature et celles de la longue transition que le monde n'ait jamais connue ont provoqué une restructuration de l'Etat qui ne correspond nullement à l'idée d'un Etat dans le sens occidental, c'est-à-dire un Etat avec une bureaucratie disciplinée, des agents fiscaux dignes, un système judiciaire neutre,...d'une part, et de l'autre, nulle n'ignore que la République Démocratique du Congo a connu toutes formes de crises politiques et son panorama politique a été gâché par des facteurs humains, la mauvaise gouvernance, les rivalités régionales, les luttes pour le pouvoir.

En effet, les différentes crises politiques, économico-sociales, rebellions constatées à l'Est et ayant conduit à des violations néfastes des droits de l'homme ont amené les Nations-Unies, la Communauté internationale, l'Union africaine et les autres acteurs à décider de convier les politiques congolais à la tenue des assises pouvant les aider à se retrouver autour d'une même table afin d'analyser, examiner ensemble les problèmes que la République Démocratique du Congo connaît et de proposer des pistes des solutions qui aideraient le pays à connaître un avenir meilleur. Pour rappel, il s'agit des assises de Lusaka, San city, Pretoria, Kampala pour négocier avec les M23, Addis-Abeba ayant débouché à l'accord d'Addis-Abeba en date du 24 Février 2013 et tout récemment les concertations nationales organisées au Pays. Parmi les résolutions prises de ces assises, l'amélioration des conditions de vie ou du social de la population congolaise, l'organisation et la tenue des élections présidentielles, législatives, sénatoriales, urbaines etc.....y figuraient et pour y arriver, une loi électorale a été votée au Parlement et promulguée par le Président de la République. ${ }^{3}$ Il s'agit de la loi $n^{\circ} 11 / 003$ du 25 Juin 2011 modifiant la loi n06/006 du 09 Mars 2006 portant organisation des élections présidentielles, législatives, provinciales, urbaines, municipales et locales.

Par ailleurs, pour permettre à tous les partis politiques de battre convenablement campagne au moment des élections et d'avoir les mêmes chances de participation aux échéances électorales, une autre loi a été votée au Parlement et Sénat et a été promulguée

1 Assistant à la Faculté de Droit de l'Université de Lubumbashi, mail: martialkakudji@yahoo.fr

2 Assistant à la Faculté de Droit de l'Université de Lubumbashi, mail: jeanmarcmutonwa@yahoo.fr

3 Loi n $11 / 003$ du 25 Juin 2011 modifiant la loi n06/006 du 09 Mars 2006 portant organisation des élections présidentielles, législatives, provinciales, urbaines, municipales et locales. 
par le Chef de l'Etat. Il s'agit de la loi n08/005 du 10 Juin 2008 portant financement public des partis politiques en République Démocratique du Congo. Selon l'esprit de ladite loi, le financement public des partis politiques dans ce pays devrait être organisé en vue de :

- Stabiliser et consolider la démocratie pluraliste par le renforcement préalable de la capacité d'action des partis politiques;

- Assurer une plus grande indépendance des partis politiques;

- Garantir l'égalité des chances entre tous les partis politiques représentés aux assemblées délibérantes par un mode de calcul simple qui repose sur le nombre de leurs élus respectifs. Le mode de calcul des crédits à allouer s'effectuant selon un coefficient de pondération variant de l'Assemblée Nationale et du Sénat aux organes délibérants locaux;

- Contribuer à la moralisation de l'activité politique par une grande transparence;

- Promouvoir la vertu de l'égalité de traitement;

- Doter les partis politiques d'un minimum de moyens pour le financement de leurs activités politiques.

Il se déduit de ce qui précède que loi $n^{\circ} 08 / 005$ du 10 Juin 2008 portant financement des partis politiques prévoit d'une part, une subvention destinée à contribuer à certaines dépenses de fonctionnement des partis politiques et, d'autre part elle constitue un moyen de financement de leurs campagnes électorales.

Parler de l'organisation des élections, de loi électorale et de financement public des partis politiques présuppose la mise en place d'une institution cadre devant organiser les élections envisagées, réguler et contrôler l'application de la loi électorale et même de veiller ou de s'assurer que les partis politiques en question et en compétition électorale ont tous bénéficié du financement public de l'Etat Congolais leur permettant de mener une campagne équilibrée et équitable. Ces exigences motivent l'adoption et la promulgation de la loi portant création, organisation et fonctionnement de la Commission électorale nationale indépendante ${ }^{4}$.

Dans la présente réflexion, nous voulons focaliser notre attention sur la mise en application des dites dispositions légales et principalement de celle relative au financement public des partis politiques. Aussi, sera-t-il question de savoir si ce financement ou ce non financement a eu de l'impact sur les résultats obtenus par les partis politiques ayant participé aux élections législatives du 28 Novembre 2011.

Comme tout travail qui se veut scientifique et qui exige une méthode, le nôtre aussi obéît à cette règle par l'utilisation de la méthode dialectique sous ses trois lois, à savoir : la loi du changement, la loi de la totalité et la loi de la contradiction en vue d'analyser et d'interpréter la loi sur le financement des partis politiques.

Avant d'aborder le fond de cette réflexion, dégageons au point qui va suivre l'esprit de la loi dont question dans la présente étude.

4 Loi organique $\mathrm{n}^{\circ} 10 / 013$ du 28 Juillet 2010 portant organisation et fonctionnement de la commission Electorale Nationale Indépendante. 


\section{I.1. L'ESPRIT DE LA LOI N08/005 DU 10 JUIN 2008 PORTANT FINANCEMENT PUBLIC DES PARTIS POLITIQUES.}

La loi portant financement public des partis politiques trouve son fondement juridique dans l'article 6 de la constitution qui dispose, d'une part, que : « Les partis politiques concourent à l'expression du suffrage, au renforcement de la conscience nationale et à l'éducation civique » et, d'autre part, que : « les partis politiques peuvent recevoir de l'Etat des fonds publics destinés à financer leurs campagnes électorales ou leurs activités, dans les conditions définies par la loi. »

Le financement dont il est question, n'est que subsidiaire, en ce sens qu'il ne vient qu'en appui aux ressources propres des partis politiques et, partant, ne peut être source d'enrichissement personnel.

Le financement public est constitué des fonds prévus aux crédits budgétaires de l'Etat. Il concerne aussi bien les dépenses couvrant les activités permanentes des partis politiques que celles consacrées à l'organisation des campagnes électorales.

Le montant de la subvention à inscrire chaque année dans la loi des finances pour contribuer aux dépenses de fonctionnement des partis politiques ne peut être ni inférieur à $0.5 \%$ ni supérieur à $1 \%$ de la totalité des recettes à caractère national revenant à l'Etat et, d'autre part, la participation de l'Etat au financement des campagnes électorales à inscrire dans la loi des finances de l'année qui suit l'organisation de chaque consultation électorale est fixée à $2 \%$ des recettes visées ci-dessus.

En vue d'éviter la complaisance et les détournements des fonds par les bénéficiaires de ce financement, la loi distingue les règles de gestion pour le fonctionnement courant de celles à suivre dans la gestion des fonds reçus aux fins de la campagne électorale.

Les subventions allouées aux partis politiques, dit la loi, sont mises à leur disposition par une commission interinstitutionnelle relevant du Ministre ayant les affaires intérieures dans ses attributions. Leur gestion obéit aux règles de la comptabilité publique et est soumise au contrôle de la cour des comptes.

Le financement des partis politiques est constitué de fonds publics prévus aux crédits budgétaires de l'Etat. Ces fonds tiennent compte des impératifs du cadrage budgétaire et ne viennent qu'en appui aux autres ressources des partis politiques ${ }^{5}$ et ce, selon les conditions et modalités données par loi elle-même. Qu'en est-il alors des conditions et modalités du financement des partis politiques.

5 Article 22 de la loi n ${ }^{\circ} 04 / 002$ du 15 Mars 2004 portant organisation et fonctionnement des partis politiques. 


\section{I.2. CONDITIONS ET MODALITES DE FINANCEMENT PUBLIC}

Les articles 1èr et 2ème de la loi sous étude disposent respectivement que : " Les partis politiques peuvent recevoir de l'Etat des fonds publics destinés à financer leurs campagnes électorales ou leurs activités, dans les conditions et selon les modalités définies par la loi. $\left.{ }^{6}{ }\right\rangle$

Eu égard à ce qui précède, il convient de donner dans les lignes suivantes les conditions prévues par la loi. Pour bénéficier des subventions de l'Etat, tout parti politique doit réunir les conditions ci-après :

- Etre régulièrement enregistré au Ministère ayant les affaires intérieures dans ses attributions;

- Avoir un siège connu et attesté par un titre de propriété ou par un contrat de bail;

- Disposer d'un compte bancaire ayant un solde créditeur d'au moins 2500000 francs congolais;

- Tenir une comptabilité régulière et disposer d'un inventaire de ses biens meubles et immeubles et produire l'attestation fiscale du dernier exercice;

- Tenir compte de la parité homme, femme, lors de l'établissement des listes électorales;

- Introduire une demande écrite à la commission interinstitutionnelle.

- La subvention est allouée aux partis politiques représentés au moins à une des assemblées délibérantes, proportionnellement au nombre de leurs élus. Il s'agit de :

- L'assemblée nationale;

- Le sénat;

- L'assemblée provinciale;

- Le conseil urbain;

- Le conseil municipal;

- Le conseil de secteur ou de chefferie.

- Enfin, les listes des élus par parti politique sont fournies par les bureaux respectifs de ces assemblées. L'Etat participe à posteriori au financement des campagnes électorales des partis politiques.

Pour bien comprendre la notion de financement des partis politiques, il est mieux de connaître le sens d'un parti politique, son rôle et ses ressources.

\section{I.3. DEFINITION D’UN PARTI POLITIQUE, ROLE ET MOYENS.}

\section{I.3.1. Définition}

Sansavoir la prétention de verser dans les détails définitionnels sur les différentes conceptions et typologies qui entourent la notion de science politique, qu'il nous soit permis de définir le terme parti politique avec Maurice DUVERGER.

L'auteur commence par définir le concept science politique qu'il considère proche à celui de parti politique. La science politique est une science du pouvoir politique entendu

6 Articles 1 et 2 de la loi n $08 / 005$ du 10 Juin 2008 portant financement public des partis politiques. 
dans le sens du pouvoir global et exercé dans toute collectivité (Groupe ou société global) c'est-à-dire le pouvoir de réaliser cette globalité, de la maintenir, de la développer, de la protéger contre les autres par opposition aux pouvoirs relatifs à chacun des secteurs dans lesquels se manifeste la collectivité en question. ${ }^{7}$

La science politique est ainsi perçue comme étant tout groupe d'individus qui, professant le même point de vue politique, s'efforce de le faire prévaloir à la fois en y ralliant le plus grand nombre de citoyens et en cherchant à conquérir le pouvoir en vue d'influencer les décisions.

\section{I.3.2. Rôle d'un parti politique et sources de financement}

Le rôle de tout parti politique est de contribuer à l'intégration de toute la communauté, de promouvoir le développement du bien être des habitants d'un pays, d'éduquer la masse partisane, de chercher à conquérir le pouvoir et de le conserver le plus longtemps possible etc. ... Pour cette raison, un parti politique doit avoir une politique qui vise l'amélioration des conditions de vie de la population et la sauvegarde d'intérêts de chaque citoyen.

En principe, un parti politique tire les moyens de sa survie et de ses activités des cotisations et dons des militants, les indemnités d'élus, les prêts et le lobbying qui constitue une pratique qui est souvent de mise aux Etats-Unis. C'est plus les indemnités des élus qui proviennent des versements en pourcentage prélevés sur les indemnités mensuelles et versés à la caisse du parti qui constituent actuellement le seul moyen de financement sûr d'un parti politique. $^{8}$

\section{LES ELECTIONS LEGISLATIVES PAR LA COMMISSION ELECTORALE NATIONALE INDEPENDANTE}

\section{II.1. De l'esprit de la loi relative aux élections législatives de 2011}

La loi électorale $n^{\circ} 06 / 006$ du 09 mars 2006 modifiée et complétée par celle $n^{\circ} 11 / 003 /$ du 25 Juin 2011 portant organisation des élections prise en application de l'article 5 de la constitution, marque une étape décisive dans le processus conduisant à des élections régulières, libres et transparentes.

La loi susdite encourage le processus démocratique et dispose que : « Le peuple congolais s'est engagé résolument dans la voie de la démocratie en adoptant sa constitution par le

7 Duverger, M., Sociologie de la Politique, P.U.F., Paris, 1979, P.180.

8 MABIALA MANTUBA NGOMA, Le financement des partis politiques, In MABIALA MANTUBA NGOMA (dir.), organisation et fonctionnement des partis politiques en République Démocratique du Congo, FKA, Kinshasa, 2004, P.39. 
référendum du 18 et 19 décembre $2005 .{ }^{9} \mathrm{Ce}$ fait constitue un signal fort pour la démocratie en République Démocratique du Congo.

En conformité avec la charte des Nations-Unies, la déclaration universelle des droits de l'homme et la charte africaine des droits de l'homme et des peuples mettent en œuvre entre autres les principes suivants :

- Le peuple est la source exclusive du pouvoir

- La volonté du peuple s'exprime par des élections régulières suivant une procédure garantissant la liberté et le secret du vote;

- Tout citoyen en âge de majorité a le droit de participer à la direction des affaires politiques du pays dans les conditions fixées par la constitution et la loi n06/006 du 09 Mars 2006, modifiée et complétée par celle $n^{\circ} 11 / 003 /$ du 25 Juin 2011. La liberté de candidature est garantie. Elle implique que des candidats indépendants, des partis politiques et regroupements politiques puissent se présenter quel que soit le mode de scrutin. Tous les candidats bénéficient d'un traitement égal de la part de l'Etat, notamment dans l'utilisation des médias,...Il convient de signaler à ce propos qu'à l'heure actuelle ce mode de scrutin a été modifié.

\section{II.2. Cadre juridique et institutionnel}

Un solide cadre juridique est essentiel pour l'administration efficace d'élections démocratiques. Le cadre juridique est composé des lois nationales qui réglementent la manière dont doit se dérouler le processus électoral. Le cadre juridique doit être conforme aux obligations de l'Etat en matière de droits de l'Homme.

La République Démocratique du Congo a ratifié un certain nombre de traités nationaux et internationaux à travers lesquels le pays s'engage à respecter certains standards clés en matière des droits de $1^{\prime}$ Homme. ${ }^{10}$ La ratification des certains textes ou instruments juridiques internationaux pour le processus électoral et le fait de leurs mise en place pour les élections par la République Démocratique du Congo, a permis que le cadre juridique du système électoral législatif comme présidentiel soit en général conforme aux normes internationales. Ce cadre a été marqué par :

1. La révision constitutionnelle de Janvier 2011 qui a introduit un seul tour pour l'élection présidentielle, un système qui est généralement déconseillé par les sociétés dominées par des tensions ethniques ou régionales;

2. La volonté de la commission électorale nationale indépendante de faire respecter à tout prix la durée du mandat présidentiel de cinq ans, qui a fait du 06 décembre 2011 la date

9 Commission électorale indépendante, Assemblée nationale et Sénat, loi nº6/006 du 09 Mars 2006 portant organisation des élections présidentielles, législatives, provinciales, urbaines, municipales et locales, P.1.

10 La constitution congolaise déclare que les traites et accords internationaux régulièrement ratifiés par la République Démocratique du Congo sont Supérieurs aux les lois nationales. 
limite pour la publication des résultats provisoires, nonobstant les difficultés techniques et logistiques pour l'élection présidentielle et celle législative.

Si la réforme constitutionnelle de Janvier 2011 qui a introduit le scrutin à un seul tour pour l'élection présidentielle est conforme au droit interne, elle n'a pas néanmoins été soumise à un référendum puisqu'elle a été approuvée à la majoritaire des trois cinquièmes des nombres de l'Assemblée Nationale et du Sénat réunis en congrès. Pourtant, cette modification de la constitution de la République Démocratique du Congo s'éloigne de la doctrine constitutionnelle générale ${ }^{11}$ selon laquelle le système électorale ferait partie du «bloc » constitutionnel. Dans ces conditions, il ne pourrait être modifié que par une procédure plus stricte que celle qui est prévue dans le cadre d'un amendement, sans pour autant avoir la rigidité de celle qui est utilisée pour l'approbation de la constitution elle-même.

La loi électorale de 2006, quoique conforme aux normes internationales, cependant a été modifiée en Août 2011, au moment où les candidats déposaient leurs dossiers de candidatures à la commission électorale nationale indépendante. Cette modification, faite au début du processus électoral et touchant des éléments importants comme la procédure du contentieux électoral, est contraire au principe de stabilité du droit électoral, lequel considère que cette stabilité est essentielle afin de non seulement garantir une bonne connaissance du cadre juridique par les acteurs politiques, mais aussi d'éviter toute manipulation du droit par les autorités en leur faveur. Par ailleurs, ces modifications de la loi électorale ont introduit de nouvelles garanties pour le scrutin tel le cas du droit des témoins des partis politiques et des candidats à réclamer une copie du procès-verbal de résultat de vote, mais elles n'ont pas comblé certains vides juridiques importants :

1. L'absence d'une procédure précise permettant à la commission électorale nationale indépendante de veiller sur la régularité du processus et des procédures spécifiques pour certains délits électoraux pouvant survenir pendant la campagne électorale (activités de campagne en dehors du délai légal, utilisation de biens ou fond publics, atteintes à la liberté de campagne);

2. La non-régulation des finances des partis politiques en l'absence de contrôle effectif pour garantir l'égalité des chances entre les candidats et aussi assurer la transparence par rapport aux comptes de campagne;

3. L'absence de dispositions claires concernant le droit des témoins à exiger des présidents de bureaux de vote la copie des résultats, la mention de leurs observations et réclamations pendant le scrutin et le dépouillement ou lorsqu'un des membres de la commission électorale nationale indépendante refuse de signer les procès-verbaux de consolidation des résultats.

11 Roland Pourtier, «Les élections de 2011 en RDC, entre cafouillage et tripatouillage », EchoGéo [En ligne], Sur le Vif, mis en ligne le 02 juillet 2012, consulté le 27 mars 2014. URL : http://echog eo.revues.org/13119; DOI : 10.4000/echogeo.13119. 


\section{II.3 Déroulement des faits pendant l'élection législative de 2011}

Certes, les élections législatives de 2011 se sont déroulées, mais non sans difficultés. La période pré-électorale a été caractérisée par des violences entre les jeunes de certains partis de la majorité présidentielle et ceux de l'opposition, l'inégalité d'accès aux médias et fermeture de certains médias à tendance d'opposition ${ }^{12}$.

En effet, l'analyse du déroulement de faits pendant cette période démontre que les retards intervenus dans la mise en place de la commission électorale nationale indépendante et dans le démarrage des opérations logistiques et techniques (enrôlement des électeurs, constitution du fichier électoral, affichage des listes d'électeurs, disponibilité et répartition du matériel électoral, désignation et formation des responsables des bureaux de vote,...) ont eu des répercutions négatives et dommageables sur le déroulement de la campagne électorale, laquelle, de ce fait, a été marquée par des incidents ayant, dans certains cas, causé des pertes en vies humaines, et troublé la conduite des opérations de vote ${ }^{13}$. Les opérations de vote proprement dites, qui ont été prolongées dans certaines localités et villes pendant trois jours et ont été marquées par des irrégularités et d'importants dysfonctionnements. Le taux de participation (58\% des électeurs c'est-à-dire un peu plus de 32 millions d'inscrits), peut en partie s'expliquer par les difficultés techniques ayant entraîné de nombreuses omissions sur les listes électorales.

D'ailleurs sur ce fait, la Mission d'Observation Electorale de l'Union Européenne a relevé que $17 \%$ des électeurs enrôlés ont dû voter sur des listes de dérogation ${ }^{14}$.

La formation des agents électoraux intervenue tardivement et rendue difficile par l'indisponibilité du matériel électoral a également été une des raisons de nombreux dysfonctionnements enregistrés. Aussi, ne pourrions-nous pas parler de la faible présence de la sécurité dans les centres de vote, la circulation des bulletins de vote avant le vote et ceux déjà cochés au moment de vote parmi le public. ${ }^{15}$ La création précipitée d'extensions des bureaux de vote a posé problème aux nombreux électeurs sans moyen de déplacement.

12 Radio.okapi.net, consulté le 22/03/2014.

13 Rapport de l'organisation de la Francophonie sur les élections présidentielles et législatives du 28 Novembre 2011 en République Démocratique du Congo.

14 Rapport final de la Mission d'observation électorale de l'union européenne sur les élections présidentielles et législatives en République Démocratique du Congo du 28 Novembre 2011.

15 Afrique.kongotimes.info., consulté sur le net le 20 Mars 2014. 


\section{PARTIS POLITIQUES AYANT PARTICIPE AUX ELECTIONS ET LA PROBLEMATIQUE DE LEUR FINANCEMENT PAR L'ETAT}

\section{III.1. De la participation aux élections des partis politiques}

D'après une étude menée, la République Démocratique du Congo compte actuellement 449 partis politiques en enregistrés au Ministère de l'intérieur ${ }^{16}$. Ces partis politiques sont tenus de se conformer aux prescrits de l'article 21 de la loi n04/002 du 15 Mars 2004 portant organisation et fonctionnement des partis politiques en République Démocratique du Congo qui exige que le compte financier d'un parti politique soit déclaré au plus tard le 31 Mars de l'exercice écoulé et lequel doit faire apparaître que le parti ne bénéficie pas d'autres sources que celles provenant des subventions éventuelles de l'Etat, cotisations, dons et legs de ses adhérents et sympathisants....

Faute d'un personnel suffisant et de la volonté politique, le Ministère de l'intérieur n'applique pas les dispositions y afférentes. D'après certains renseignements recueillis, il s'avère que les partis politiques ne respectent guère cet article. ${ }^{17}$ Et cela, ne permet pas au Ministère de faire rapport sur ces partis ni de les contrôler. Pour des raisons propres à la présente étude, nous donnerons seulement la liste des partis phares ayant participé au législatif de 2011 et leur tendance politique. Par illustration, il s'agit des partis suivants : le Parti du Peuple pour la Reconstruction; Parti Lumumbiste Unifié; Mouvement Social pour le Renouveau; Union Nationale des Démocrates Chrétiens; Union Nationale des Démocrates Fédéralistes; Alliance pour le Renouveau du Congo, Parti de la Révolution du Peuple etc.... qui sont de la majorité présidentielle d'une part, et de l'autre, l'Union pour la Démocratie et le Progrès Social; Mouvement de Libération du Congo; Rassemblement Congolais pour la Démocratie; Union pour la Nation Congolaise; Convention des Démocrates Chrétiens; Union des Démocrates Mobutistes etc.....de la tendance opposition.

III.2. Problématique du financement des partis politiques par l'Etat et son impact sur les résultats du législatif

La loi portant financement public des partis politiques prévoit que les partis politiques peuvent recevoir de l'Etat des fonds publics destinés à financer leurs campagnes électorales ou leurs activités, bien entendu, dans les conditions définies par la loi. Aussi, précise-t-elle, non seulement la marge de la subvention en terme de pourcentage, mais aussi stipule que le financement doit être constitué des fonds prévus aux crédits budgétaires de l'Etat.

16 NOEL OBOTELA RASHIDI et Alii, Les partis politiques congolais en question Plaidoyer pour des structures durables et organisées - Etude par le Centre d'Etudes politiques et la fondation Konrad Adenauer, Etude menée suivant le projet IB 13 - 002, Juillet - Décembre 2013 à Kinshasa.

17 Ces informations ont été recueillies, le 6 Décembre 2013, à la Direction des services généraux du secrétariat général au Ministère de l'Intérieur, sécurité, décentralisation et affaires coutumières, en charge des Relations avec les partis politiques. 
De part l'analyse de cette loi, on comprend que l'Etat Congolais avait le devoir de financer les partis politiques pour leurs campagnes électorales et leurs activités, mais ce financement doit être prévu aux crédits budgétaires.

Par ailleurs, l'étude de la loi des finances de l'exercice 2010-2011 démontre que cette loi n'avait pas prévu une rubrique propre destinée au financement des partis politiques néanmoins, dans cette loi, il a été prévu de manière globale une rubrique pour les élections. Ceci constitue déjà un problème d'application de la loi de financement public des partis politiques en ce sens que l'institution habilitée à adopter cette loi de finances et à la voter, regorgeait les futurs candidats aux législatifs de 2011 mais qui, malheureusement, n'y avaient pas pensé. Mais il sied de stigmatiser que le fait que le Parlement ait prévu dans la loi des finances une rubrique pour le financement des élections, cela présuppose que même les partis politiques et leurs campagnes électorales devraient être financés et ce, parce que personne ne pouvait ignorer que les élections impliqueraient les dépenses pour les partis politiques et leurs campagnes.

Au moment des campagnes électorales, le Gouvernement Congolais s'est déclaré incapable de financer les partis politiques pour leurs campagnes électorales et leurs activités par ce que ne disposant pas des moyens conséquents. Au même moment, relevons le fait que le bloc de la majorité présidentielle regorgeait à son sein des candidats ayant occupé des grands postes de responsabilité au sein des institutions étatiques en l'occurrence le poste d'Ambassadeurs itinérants du Président de la République et autorité morale de la coalition, Ministres, Mandataires publics et des entreprises publiques, Gouverneurs et vices Gouverneurs des provinces. Ceux-ci de part les postes qu'ils s'occupaient, disposaient des ressources suffisantes au détriment des candidats indépendants et ceux de l'opposition. Le non financement public des partis politiques a eu, sans doute, un impact sur les résultats du législatif de 2011 donnés par la Commission électorale nationale indépendante.

A l'issue des élections législatives du 28 novembre 2011 l'on pouvait noter les résultats ci-après : le Parti du Peuple pour la Reconstruction et la Démocratie (P.P.R.D.) s'était imposé comme la première force en termes de poids politique à l'Assemblée Nationale avec 57 députés élus, selon la publication des résultats provisoires partiels des législatives 2011. S'il n'arrivait pas à rafler la majorité absolue, à lui seul, il aura néanmoins à composer avec d'autres partis pour former la majorité.

L'Union pour la Démocratie et le Progrès Social (U.D.P.S) d'Etienne TSHISEKEDI avait remporté 34 sièges, suivie du Parti du Peuple pour la Paix et la Démocratie (P.P.P.D) avec 27 députés élus et du Mouvement Social pour le Renouveau (M.S.R.) avec 25 députés élus. Quant au Mouvement de la Libération du Congo (M.L.C.), il a eu 19 députés élus et le Parti Lumumbiste (P.A.L.U.) 16 députés élus. L'Union pour la Nation Congolaise (U.N.C.) de Vital KAMERHE a, quant à elle, fait une entrée encourageante dans le trio de tête des partis de l'opposition à l'Assemblée nationale. Les formations politiques comme l'Alliance pour le Renouveau du Congo (A.R.C.) et la Convention des Congolais unis (C.C.U.) ont respectivement obtenues 14 et 5 députés élus. 
Sur 18.865 candidats enregistrés, 18.365 sont sensés perdre et sont donc à fortiori ou à raison des potentiels contestataires. Toutefois, il y a à regretter que la plupart des corrupteurs, dit le journal « la Prospérité », que se sont malheureusement recrutés parmi les 18.865 candidats à la députation nationale ${ }^{18}$. Nous ne disons pas que tous les 18.865 candidats se sont compromis. Ce fléau de corruption, mentionné par le journal « la Prospérité », mérite d'être combattu pour donner des chances à ceux qui ont réellement mérité la confiance du souverain primaire.

Les tensions et violences électorales ont été utilisées comme armes de compétition par les acteurs politiques Congolais. En voici les cas : dans la nuit du 5 au 6 septembre 2011, à Kinshasa, un bureau du P.P.R.D. a été incendié. Suite à cet incident, des manifestants du P.P.R.D., qui auraient été aidés par des agents de la Police Nationale Congolaise, ont pillé le quartier général de l'U.D.P.S. à Kinshasa; la même nuit, les bureaux de la Radio Lisanga Télévision (RLTV), une station radio appartenant à l'opposition soutenant Etienne TSHISEKEDI, ont été incendiés; lors d'affrontements entre des militants de l'U.D.P.S. et du P.P.R.D. à Kinshasa, le 29 septembre 2011, un caméraman de la Radio France Outre-mer aurait été sévèrement battu par des militants du P.P.R.D; le 8 janvier 2011, à Lubumbashi, province du Katanga, deux activistes des droits de l'homme, travaillant pour deux Organisations Non Gouvernementales, ont été menacés d'arrestation par deux éléments non identifiés des forces de sécurité en tenue civile.

A Kinshasa, deux membres éminents d'une organisation nationale des droits de l'homme ont reçu des menaces de mort les 1er et 2 février 2011. Ces menaces ont suivi une conférence de presse organisée par les victimes au cours de laquelle elles ont dénoncé la révision constitutionnelle et l'intolérance vis-à-vis de l'opposition.

Le silence observé dans les milieux politiques et diplomatiques et même religieux des actes de violences provoqués par les acteurs politiques, qui ont émaillé les élections couplées du 28 novembre 2011, inquiétait tout observateur épris de justice.

Ces actes de violences étaient perpétrés contre les installations ou les agents de la Commission Electorale Nationale Indépendante par les candidats ou leurs militants et des cas avérés de substitution des résultats de certains candidats dans les Centres locaux de compilation des résultats ont été constatés (C.L.C.R.) ${ }^{19}$.

Eu égard à ce qui précède et d'après nos investigations, ces résultats du législatif se justifieraient par la non application de la loi portant financement des partis politiques. Chaque parti politique a financé sa campagne électorale. Hélas! Les choses sont simples. Le parti au pouvoir (P.P.R.D.) s'en est parti en campagne électorale avec les autres partis politiques

18 « La prospérité » in législatives, 2011 le P.P.R.D et l’U.D.P.S en fête, 30 janvier 2012.

19 Rapport Annuel 2011 sur la situation globale des Droits de l'homme en R.D.C. par le Secrétariat Exécutif National du Réseau National des ONGS des Droits de l'homme de la R.D.C./ National Net work of Congolese Human Right NGO (RENADHOC) sous le thème : «Problématique des Droits de l'homme et des élections présidentielles et législatives en R.D.C.: Duel entre l'alternance et le statu quo.». 
sans avoir la même base rationnelle, de gros moyens financiers contre ceux qui en ont moins vivant ne fut ce que des cotisations des membres fondateurs des partis.

Cet état de choses a encouragé le P.P.R.D. a encore crée d'autres partis satellites qui ne sont plus à la une mais qui n'existent que de nom et surtout d'autres embranchement dénoncés par les partis d'opposition avant la campagne électorale. Il s'agit du Parti du Peuple pour la Paix et la Démocratie (P.P.P.D.) et du Mouvement pour l'Intégrité du Peuple (M.I.P.) qui serait dirigé avant par Collette TSHOMBA. Aujourd'hui, tous les élus de ces deux partis politiques sont mués en P.P.R.D. Conséquence, le P.P.R.D. a à lui seul une nonantaine des députés sans compter ceux de ses partis satellites et de la majorité présidentielle. Et, les calculs deviennent faciles pour la mise en place des institutions issues des urnes.

\section{CONCLUSION}

La situation en République Démocratique du Congo nécessite un changement profond et radical en vue de l'amélioration des conditions de vie de la population susceptibles d'assurer son épanouissement et son développement; ce qui doit être l'œuvre de l'autorité qui édicte les lois et les faits appliquer.

Le changement nécessite la prise de conscience de chaque individu dans l'accomplissement de ses attaches quotidiennes en termes de sa profession exercée. L'organisation des élections traduit la volonté d'un pays qui se veut un Etat de droit et démocrate et lequel, doit respecter et appliquer ses propres lois pour éviter tout soupçon ou forme des contestations surtout dans le domaine électoral. Voilà pourquoi les dirigeants politiques doivent militer en faveur du changement en vue d'assurer à la population le bien-être collectif.

Le non financement des partis politiques par l'Etat Congolais a été aussi à la base d'échecs de nombreux partis politiques dont leurs candidats ne juraient que par ce financement pour se doter des moyens qui seraient égaux à ceux de la majorité dont la plupart de candidats disposaient déjà des moyens de part les postes qu'ils occupaient dans les institutions de la République. Les investigations menées démontrent que les modalités pratiques d'application de la réglementation sur le financement de la campagne électorale, pourtant prévue dans la loi « portant financement public des partis politiques » adoptée en juin 2008, n'existent pas.

Ainsi, nous recommandons que cette question trouve rapidement une réponse qui permettrait d'éviter des inégalités entre partis politiques ou candidats. Les partis politiques doivent se conformer à la loi et aux exigences y afférentes en vue de bénéficier du financement qui, du reste, doit être compris comme subsidiaire.

Le financement des partis politiques devrait servir d'équilibre aux candidats pendant la campagne électorale. Tous les candidats devraient avoir les mêmes moyens avant de s'engager dans la campagne électorale. Cependant, à l'exception des sympathisants qui battaient campagne pour leurs candidats, s'était le tour des candidats eux-mêmes de se présen- 
ter devant les électeurs pour se faire connaître, présenter leurs plans d'actions, se faire aimer ou se faire attirer la sympathie afin que les électeurs votent pour eux.

Pour cela, il fallait parcourir les coins et recoins de villes et villages de sa circonscription électorale en organisant des émissions radiotélévisées, des réunions électorales restreintes ou de grands rassemblements publics, en mettant les affiches, les spots publicitaires et autres, faute de moyens matériel et financier, beaucoup de candidats n'ont pas réussi à le faire. Ne serait pas de faits anti-démocratiques, car la démocratie implique le principe de l'égalité pour tous.

S'agissant de l'éradication de la corruption décriée par plusieurs candidats de l'opposition lors de l'élection législative, retenons que le financement des partis politiques ne peut pas mettre fin à la corruption lors des élections. Néanmoins, ce financement pourrait contribuer à la diminution du taux de corruption pendant la campagne électorale et le jour de scrutin.

Aux députés élus et à qui incombent le pouvoir de voter la loi des finances, de veiller, avant l'adoption de celle-ci, à insérer une rubrique propre au financement des partis politiques. Ceci servira de contrainte dans le chef du Gouvernement de financer les partis politiques.

La loi portant financement public des partis politiques doit recevoir application en République Démocratique du Congo pour éviter toute forme de partialité. Pour cela, il faut mettre en marche et en synergie tous les acteurs impliqués dans le processus électoral. Au Parlement de contrôler l'application de la loi et d'ailleurs, pour le législatif de 2011, il devrait interpeler ou contrôler le Ministère de l'intérieur pour savoir pourquoi le Gouvernement n'avait pas financé les partis politiques.

Etant donné que le peuple congolais est encore au stade qu'on pourrait considérer d'immaturité politique qui entraine l'ignorance des droits et devoirs fondamentaux de tout citoyen, une importante marche de progrès reste à faire, en l'occurrence en matière de contrôle inter-pouvoirs et du respect de la règle de droit(de la loi) ainsi que de la connaissance de celle-ci par le peuple.

Car comme le dit PIERRE BOURDIEU ${ }^{20}$, Sociologue Français d'heureuse mémoire, la lutte politique est essentiellement la lutte des discours. Elle atteint son paroxysme pendant la campagne électorale car c'est le moment où différents acteurs politiques qui vont à la conquête des positions de pouvoir cherchent à mobiliser la plus grande frange de l'opinion en leur faveur par le mécanisme de la violence symbolique. La conquête d'un électorat exige des moyens financiers suffisants.

20 André LUBANZA M., Elections et construction des identités politiques en R.D.C., CEROPIC, S.E., S.D. 


\section{REFERENCES BIBLIOGRAPHIQUES}

1. La constitution de la République Démocratique du Congo du 18 Février 2006

2. Loi $n^{\circ} 11 / 003$ du 25 Juin 2011 modifiant la loi n06/006 du 09 Mars 2006 portant organisation des élections présidentielles, législatives, provinciales, urbaines, municipales et locales

3. Loi organique $\mathrm{n}^{\circ} 10 / 013$ du 28 Juillet 2010 portant organisation et fonctionnement de la commission Electorale Nationale Indépendante

4. Article 22 de la loi $n^{\circ} 04 / 002$ du 15 Mars 2004 portant organisation et fonctionnement des partis politiques

5. Commission électorale indépendante, Assemblée nationale et Sénat, loi n ${ }^{\circ 06 / 006 ~ d u}$ 09 Mars 2006 portant organisation des élections présidentielles, législatives, provinciales, urbaines, municipales et locales

6. Articles 1 et 2 de la loi $n^{\circ} 08 / 005$ du 10 Juin 2008 portant financement public des partis politiques

7. ANDRE LUBANZA M., Elections et construction des identités politiques en R.D.C., CEROPIC, S.E., S.D.

8. DUVERGER, M., Sociologie de la Politique, P.U.F., Paris, 1979, P.180

9. MABIALA MANTUBA NGOMA, « Le financement des partis politiques », In MABIALA MANTUBA NGOMA (dir.), organisation et fonctionnement des partis politiques en République Démocratique du Congo, FKA, Kinshasa, 2004

10. NOEL OBOTELA RASHIDI et Alii, Les partis politiques congolais en question Plaidoyer pour des structures durables et organisées - Etude par le Centre d'Etudes politiques et la fondation Konrad Adenauer, Etude menée suivant le projet IB 13 - 002, Juillet - Décembre 2013 à Kinshasa

11. ROLAND POURTIER, "Les élections de 2011 en RDC, entre cafouillage et tripatouillage », EchoGéo [En ligne], Sur le Vif, mis en ligne le 02 juillet 2012, consulté le 27 mars 2014. URL : http://echogeo.revues.org

12. Rapport du secrétariat de la Direction des services généraux du secrétariat général au Ministère de l'Intérieur, sécurité, décentralisation et affaires coutumières, en charge des Relations avec les partis politiques, consulté le 6 Décembre 2013

13. Rapport de l'organisation de la Francophonie sur les élections présidentielles et législatives du 28 Novembre 2011 en République Démocratique du Congo

14. Rapport final de la Mission d'observation électorale de l'union européenne sur les élections présidentielles et législatives en République Démocratique du Congo du 28 Novembre 2011

15. Radio.okapi.net, consulté le 22/03/2014

16. Afrique.kongotimes.info., consulté sur le net le 20 Mars 2014

17. « La prospérité » in législatives, 2011 le P.P.R.D et l'U.D.P.S en fête, 30 Janvier 2012 